\title{
Detection and sampling methods for isolation of Candida spp. from oral cavities in diabetics and non-diabetics
}

\section{Sanja MATIĆ PETROVIĆ(a) Milena CIMBALJEVIĆ(a) Milena RADUNOVIĆ(b) Jovana KUZMANOVIĆ PFIĆER ${ }^{(c)}$ Aleksandra JOTIĆ(d) Ana PUCAR(a)}

\footnotetext{
(a) University of Belgrade, School of Dental Medicine, Department of Oral Medicine and Periodontology, Belgrade, Serbia.

(b) University of Belgrade, School of Dental Medicine, Department of Microbiology and Immunology, Belgrade, Serbia.

(c) University of Belgrade, School of Dental Medicine, Department of Medical Informatics and Statistics, Belgrade, Serbia.

(d) University of Belgrade, Faculty of Medicine, Clinic for Endocrinology, Diabetes and Metabolic Disorders, Belgrade, Serbia
}

Declaration of Interests: The authors certify that they have no commercial or associative interest that represents a conflict of interest in connection with the manuscript

Corresponding Author:

Sanja Matic Petrovic

E-mail: sanjamatic@gmail.com

DOI: 10.1590/1807-3107BOR-2015.vol29.0077

Submitted: Nov 07, 2014

Accepted for publication: Feb 17, 2015

Last revision: May 14,2015

\begin{abstract}
The purpose of this study was to detect Candida spp. on the tongue and in the subgingival sites in healthy and type 2 diabetes (T2D) patients with chronic periodontitis $(\mathrm{CP})$, and to compare the accuracy of sampling methods. This study included 131 patients divided into four groups: healthy control (group A), nondiabetics + CP (Group B), diabetics with good metabolic control $+\mathrm{CP}$ (group $\mathrm{C}$ ) and diabetics with poor glycoregulation + CP (Group D). Cotton swab samples from tongue and subgingival samples were obtained from each patient with help of sterile paper points and a sterile curette. Swab cultures were made on Sabouraud dextrose agar. The number of CFUs was counted. The sampling methods for subgingival plaque were compared by Receiving Operator Curve (ROC). The presence of Candida spp. on the tongue was statistically significant among groups (group $\mathrm{D}$ vs. others three groups: $\chi^{2}: \mathrm{p}<0.005$ for each group). Positive findings of subgingival Candida spp. did not differ among the groups. There were no significant differences in the quantification of Candida spp., neither on the tongue, nor in the subgingival samples. $17.2 \%$ of diabetic patients revealed the presence of Candida spp. in the subgingival samples, with negative finding on tongue. There was a significant difference in the sampling methods for subgingival plaque $(p=0.000)$. Candida spp. is more prevalent on the tongue of diabetics. The sampling of subgingival plaque by a sterile curette is more accurate than with paper points. Subgingival plaque may represent a reservoir of commensals. It is necessary to standardize the sampling of subgingival plaque.
\end{abstract}

Keywords: Diabetes Mellitus, Type 2; Chronic Periodontitis; Candida; Periodontal Pocket.

\section{Introduction}

Type 2 diabetes mellitus (T2D) is a chronic metabolic disorder that leads to progressive defects in insulin secretion based on insulin resistance. ${ }^{1}$ For thirty years periodontitis has been acknowledged as the sixth chronic complication of diabetes. ${ }^{2,3}$ Candida infections are chronic opportunistic infections related to diabetic patients. The presence of Candida spp. in oral cavities of diabetics varies between $50-80 \%{ }^{4,5,6,7}$ Yeasts commonly inhabit tongue, palate and buccal mucosa, and it has recently been found in the subgingival sites. ${ }^{8}$ The periodontium may represent a reservoir of opportunistic microorganism, especially in immunocompromised 
patients. ${ }^{9}$ The presence of yeasts in subgingival sites was examined in relationship to general health and periodontal status. It varies between $30-50 \%$ in the case of diabetics. ${ }^{4,70}$ Yeasts ${ }^{11}$ and viruse ${ }^{12}$ could have a significant role in the pathogenesis of periodontal diseases. The immunological response around hyphae of Candida spp. is similar to the response to periopathogens of bacterial origin, and consists of chronic mononuclear inflammatory cells with sporadic neutrophil leucocytes. ${ }^{11}$ The potential role of yeasts in the pathogenesis of periodontitis is especially important for diabetic patients, because antibiotics are commonly used in the treatment of periodontitis.

The purpose of this study was to detect Candida spp. on the tongue and in subgingival sites in healthy and T2D patients with chronic periodontitis (CP), and to compare the accuracy of sampling methods.

\section{Methodology}

\section{Subjects, Ethical approval}

This cross-sectional study was approved by the Ethical Committee of the School of Dentistry, University of Belgrade (Ethics Approval no. 36/8). It included 131 patients divided into four groups. Group A $(n=35)$ consisted of healthy volunteers without clinical signs of CP. Group B $(n=30)$ consisted of healthy subjects diagnosed with CP. Group C $(n=26)$ included T2D patients with good glycoregulation and diagnosed $C P$ and group $D(n=40)$ consisted of T2D patients with poor metabolic control and diagnosed $\mathrm{CP}$.

\section{Inclusion and exclusion criteria}

The exclusion criteria were: presence of any disease except T2D, aggressive periodontitis, usage of medication that might affect the periodontium, e.g. corticosteroids, antibiotics, antiseptics, history of oral candidiosis treatment, pregnancy, lactation and periodontal treatment in the last 1.5 year.

\section{Anamnesis data and biochemical/hematological analysis}

Self-reported information about blood type, everyday intake of sweets and smoking habits were recorded. According to the blood type, patients were divided into $\mathrm{O}$ vs. $\mathrm{A}+\mathrm{B}+\mathrm{AB}$ blood type. ${ }^{13}$ Patients were classified according to their smoking status as "non-smokers" and "smokers". Fasting plasma glucose levels (FPG), glycated hemoglobin $\left(\mathrm{HbA}_{1} \mathrm{c}\right)$, hematological parameters (RBC, Hgb, HCT, MCV, MCH, MCHC, RDW) and sedimentation rate were measured.

\section{Diagnosis of CP and T2D}

T2D was diagnosed according to the criteria of the American Diabetes Association ${ }^{1}$ by measuring glycaemia during $2 \mathrm{~h} 75 \mathrm{~g}$ oral glucose tolerance test (OGTT), as well as $\mathrm{HbA}_{1} \mathrm{c}$ values. Nondiabetics exhibited normal parameters on OGTT and $\mathrm{HbA}_{1} \mathrm{c}<6.5 \%$. Glycoregulation was classified, according to $\mathrm{HbA}_{1} \mathrm{c}$, as satisfactory $\left(\mathrm{HbA}_{1} \mathrm{c} \leq 7.5 \%\right)$ and as with poor metabolic control $\left(\mathrm{HbA}_{1} \mathrm{c}>7.5 \%\right)$.

Full mouth clinical examinations were performed at six sites per tooth and evaluated on each tooth in order to access periodontal parameters: plaque index-Silness Loe (PI), dichotomous bleeding on probing (BOP), probing pocket depth (PPD) and clinical attachment loss (CAL). Two calibrated doctors performed examinations using periodontal probe (XP 23/UNC-15, Hu-Friedy, Chicago, USA). Periodontitis was diagnosed if subject exhibited CAL $>1 \mathrm{~mm}$ and PPD $>3 \mathrm{~mm}$ at least at three sites in two quadrants. ${ }^{14,15}$ Patients without clinical signs of periodontitis exhibited $\mathrm{PPD}<3 \mathrm{~mm}$ and $\mathrm{CAL}=0 \mathrm{~mm}$.

\section{Sample collection and cultivation}

Samples were collected a day after periodontal examination. Oral swabs were collected by swabbing ten times from the dorsum of the tongue with the help of a dry sterile cotton stick. Swab cultures were immediately inoculated on Sabouraud dextrose agar (SDA) (Oxoid, Basingstoke, UK). The tooth with deepest PPD was isolated by means of sterile cotton rolls and the supragingival plaque was removed by using sterile gauze and a curette. Two sterile paper points with a size of 30 were placed into the pocket for 30 s until a mild resistance appeaed. Paper points contaminated by blood were not included in the analysis. Subgingival samples were obtained from the same pocket by means of a sterile curette (S4L/4R SS G.Hartzell\&Son, Concord, California). Both subgingival samples were 
inoculated in sterile plastic tubes containing $1 \mathrm{~mL}$ of Sabouraud dextrose broth (Oxoid, Basingstoke, UK). The plastic tubes were vortexed for $1 \mathrm{~min}$ and $20 \mathrm{ml}$ of suspended broth was streaked on SDA. Samples were inoculated at $37^{\circ} \mathrm{C}$ for $48 \mathrm{~h}$. The cultural and microscopic qualities of the yeasts were examined, and the germ-tube production test, as well as the carbohydrate and potassium nitrate assimilation tests, were performed when needed. After incubation, one calibrated microbiologist counted the growth density and number of Colony Forming Units (CFUs). The yeast growth density from tongue samples was defined as rare, medium or dense. The number of CFUs was measured for samples taken by means of paper points. Depending on the $\mathrm{CFU} / \mathrm{ml}$ there were defined three groups: 1: < 500, 2: $500-2500$ and 3: > 2500.

\section{Statistical analysis}

Statistical analyses were carried out by using SPSS 18.0 software package for Windows (SPSS inc., Chicago, USA) and MedCalc for Windows, version 13.3.30 (MedCalc Software, Mariakerke, Belgium) for the Receiving Operators Curve (ROC) analysis.

Descriptive data were presented as Mean \pm SD or the percentage for discrete measures. $t$-test and One Way ANOVA were used for normally distributed data. Non-parametric data were analyzed using by using the Kruskall-Wallis and Mann-Whitney test. Categorical variables were compared using the Chi
Square Test $\left(\chi^{2}\right)$. The relationship between CFU and clinical parameters was determined by Spearman's correlation coefficient. The linear regression model was used to determine predictors of the presence of Candida spp. ROC analysis was carried out in order to compare sampling methods for subgingival plaque collection for isolation of Candida spp. Differences were considered significant when $\mathrm{p}$-value was $<0.05$.

\section{Results}

Demographic, clinical and biochemical data are presented in Table 1. Groups were matched by age, gender and smoking status.

Differences between groups $C$ and $D$ were observed for HbA1c (Mann-Whitney, $\mathrm{p}=0.000$ ) and FPG (Mann-Whitney, $\mathrm{p}=0.000$ ). Hematological parameters did not differ between groups. Groups were matched according to their blood type $\left(\chi^{2}, p=0.541\right)$.

PI differed between groups (Table 2). BOP was different between group $\mathrm{A}$ and other groups (Bonferroni: B vs. $\mathrm{C}=\mathrm{B}$ vs. $\mathrm{D}=\mathrm{C}$ vs. $\mathrm{D}: \mathrm{p}=1.000$ ). PPD did not differ significantly between groups $B, C$ and $\mathrm{D}$ (Bonferroni: $\mathrm{B}$ vs. $\mathrm{C}=\mathrm{B}$ vs. $\mathrm{D}=\mathrm{C}$ vs. $\mathrm{D}, \mathrm{p}=1.000$ ).

Positive finding of Candida spp. on tongue were found in 38/131 (27.3\%) patients. The presence of Candida spp. on tongue was significantly higher in group $\mathrm{D}\left(\mathrm{X}^{2}\right.$ : group A vs. D: $p=0.033$, B vs. D: $p=0.007, C$ vs. D: $p=0.046$ ) (Figure 1). When comparing Candida spp. findings on

Table 1. Demographic and biochemical data of patients.

\begin{tabular}{|c|c|c|c|c|c|}
\hline & Group A & Group B & Group C & Group D & p-value \\
\hline Age & $43.57 \pm 3.389$ & $47.07 \pm 10.869$ & $48.31 \pm 6.851$ & $47.55 \pm 7.527$ & 10.056 \\
\hline $\operatorname{Sex}(m / f)$ & 14 (28.6\%) / $21(71.4 \%)$ & $14(40 \%) / 16(60 \%)$ & $14(53.8 \%) / 12(46.2 \%)$ & $26(65 \%) / 14(35 \%)$ & ${ }^{2} 0.164$ \\
\hline Smokers N(\%)/ Nonsmokers N(\%) & $8(22.9 \%) / 27(77.1 \%)$ & $11(36.7 \%) / 19(63.3 \%)$ & $8(30.8 \%) / 18(69.2 \%)$ & $10(25.0 \%) / 30(75.0 \%)$ & ${ }^{2} 0.606$ \\
\hline $\mathrm{FPG}(\mathrm{mmol} / \mathrm{l})$ & $4.65 \pm .539$ & $4.73 \pm 0.624$ & $7.49 \pm 1.875$ & $11.247 \pm 4.061$ & ${ }^{3} 0.000$ \\
\hline $\mathrm{HbAlc}(\%)$ & $4.81 \pm 0.623$ & $4.86 \pm 0.635$ & $7.09 \pm 0.578$ & $10.84 \pm 1.366$ & ${ }^{3} 0.000$ \\
\hline $\operatorname{RBC}\left(x 10^{12}\right)$ & $4.53 \pm .467$ & $4.68 \pm .703$ & $4.58 \pm .363$ & $4.69 \pm .601$ & 10.587 \\
\hline $\mathrm{Hgb}$ & $137.50 \pm 10.276$ & $138.43 \pm 12.263$ & $138.88 \pm 11.669$ & $137.19 \pm 14.731$ & ${ }^{3} 0.883$ \\
\hline $\mathrm{HCT}(\mathrm{I} / \mathrm{I})$ & $0.41 \pm .0538$ & $0.43 \pm .709$ & $0.41 \pm .032$ & $0.42 \pm .064$ & ${ }^{3} 0.423$ \\
\hline MCV (fl) & $91.52 \pm 7.516$ & $91.72 \pm 7.755$ & $89.22 \pm 3.520$ & $89.28 \pm 10.486$ & ${ }^{1} 0.077$ \\
\hline $\mathrm{MCH}(\mathrm{pg})$ & $30.71 \pm 2.243$ & $30.30 \pm 2.613$ & $29.58 \pm 3.667$ & $28.98 \pm 3.062$ & ${ }^{3} 0.060$ \\
\hline $\mathrm{MCHC}(\mathrm{g} / \mathrm{l})$ & $333.88 \pm 29.170$ & $329.66 \pm 28.846$ & $339.01 \pm 14.495$ & $327.49 \pm 22.803$ & 10.196 \\
\hline RDW(\%) & $14.26 \pm 1.501$ & $14.28 \pm 1.64$ & $13.79 \pm 1.546$ & $14.68 \pm 1.789$ & 10.170 \\
\hline
\end{tabular}

All values are presented as Mean \pm SD.

${ }^{1}$ One Way ANOVA; ${ }^{2}$ Pearson Chi Square Test; ${ }^{3}$ Kruskal-Wallis; ${ }^{4}$ Independent Sample t-test. 
口 Positive finding of candida spp. on tongue

口 Positive finding of candida spp. in subgingival samples

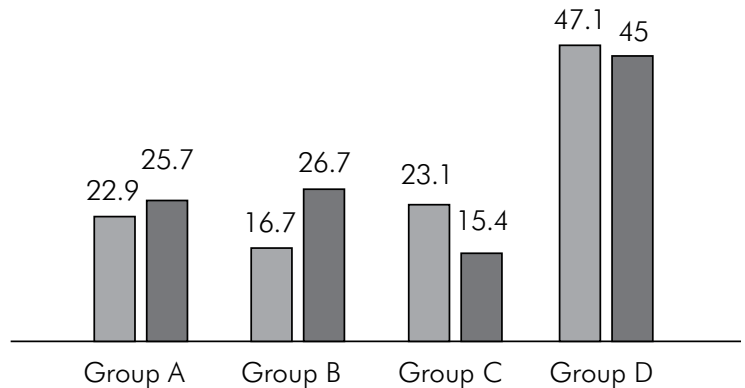

Figure 1. Presence of Candida spp. on tongue and in subgingival samples.

tongue of non-diabetics (20.3\%) and diabetics (37.9\%), a statistical difference was observed $\left(\chi^{2}, p=0.028\right)$.

The quantification of Candida spp. on the tongue did not differ between groups. The univariate logistic regression model was applied, in order to identify parameters that could predict positive finding of Candida spp. on tongue. Age, gender, blood type, everyday intake of sugars, smoking habits, number of teeth, diabetes duration, treatment mode, FPG level, HbA1c, RBC, Hgb, MCV, HCT, MCH, MCHC and RDW were analyzed as potential predictors. There was found no predictor for the positive finding of yeasts on tongue.

The subgingival findings of Candida spp. were positive in $41 / 131$ (29.50\%). There was no difference in the presence of yeast in subgingival sites between groups $\left(\chi^{2}, p=0.060\right)$ (Figure 1 ).

There was no relation between presence of subgingival Candida spp. and clinical periodontal parameters. The quantification of subgingival Candida spp. was not different between groups.

In the case of diabetic patients, there was a positive correlation between the presence of subgingival Candida spp. and HbA1c (Spearman correlation coefficient, $r=0.276, p=0.025)$.

Logistic regression analysis did not identify any parameter that could predict the presence of Candida spp. in subgingival samples.

18/131 (12.9\%) patients presented negative Candida spp. findings on the tongue and positive findings in subgingival samples.
There was a statistical difference regarding sampling methods for subgingival plaque collection and yeast detection (Table 3). The ROC curve was used to compare the diagnostic techniques of both collecting methods. The referent sampling method was by means of a sterile curette. There was a difference between methods $(p=0.000)$. Sensitivity was 0.576 and specificity was 0.919 . The area under the curve was 0.747 . Asymptomatic $95 \%$ Confidence Interval was $0.638-0.857$ (Figure 2).

\section{Discussion}

The proposed microbiological etiologies of the periodontal disease have been changing for decades. There is increasing evidence about the involvement of microorganisms other than bacteria (e.g. viruses ${ }^{11}$ and yeasts ${ }^{8,12}$ ) in the pathogenesis of periodontal disease.

Candida spp. is a common oral saprophyte. Yeast may form biofilm, which is an essential strategy for their survival in oral milieu. ${ }^{16,15}$ Beside biofilm formation, this genus is able to produce exoenzimes, proteinases and metabolites in order to adhere to epithelial cells and inhibit the function of polymorphonuclears. ${ }^{17,18,19}$ It can be isolated in about $50 \%$ of healthy population without clinical signs of infection..$^{13}$ In the case of diabetics, this prevalence is higher.

The prevalence and quantification of Candida spp. on tongue and subgingival samples were examined in diabetic and non-diabetic patients. To the best of our knowledge, this is the first study that examined patients with a clinically healthy periodontium, subjects diagnosed with periodontitis and diabetics. In our study, the overall prevalence of Candida on the tongue was $27.3 \%$. The most frequent finding was in a group of poorly controlled diabetics. Differences in the prevalence of Candida spp. on tongue in the case of diabetic patients is in accordance with other studies, ${ }^{6,20,21}$ but the percentage $(37.9 \%)$ was lower compared to other studies, where findings of Candida on tongue varied between $59-77 \%$. ${ }^{4,5,7}$ The quantification of yeast growth on tongue did not differ between groups, which is contrary to other studies ${ }^{6,22}$ probably because denture wearers were included and sampling methods were different.

Candida spp. was a commonly occurring microorganism in samples from subgingival sites. 
Table 2. Clinical periodontal parameters.

\begin{tabular}{lccccc}
\hline Clinical parameter & Group A & Group B & Group C & Group D & -value \\
\hline PI & $0.82 \pm 0.423$ & $1.72 \pm 0.767$ & $2.65 \pm 0.458$ & $2.23 \pm 2.32$ & 10.000 \\
BOP $(\%)$ & $39.61 \pm 19.273$ & $62.057 \pm 24.288$ & $64.24 \pm 24.043$ & $64.41 \pm 28.86$ & 20.000 \\
PPD $(\mathrm{mm})$ & $2.02 \pm 0.524$ & $2.89 \pm 0.944$ & $2.85 \pm 0.932$ & $2.69 \pm 0.756$ & 20.000 \\
CAL $(\mathrm{mm})$ & 0 & $3.56 \pm 2.142$ & $3.98 \pm 1.947$ & $4.12 \pm 2.104$ & 10.661 \\
\hline
\end{tabular}

All values are presented as Mean \pm SD.

${ }^{1}$ Kruskal-Wallis Test; ${ }^{2}$ One Way ANOVA.

Table 3. Comparison of sampling methods for subgingival plaque collection.

\begin{tabular}{|c|c|c|c|c|}
\hline \multirow{2}{*}{ Paper point method } & \multicolumn{2}{|c|}{ Sterile curette method } & \multirow{2}{*}{ Total } & \multirow{2}{*}{$p$-value } \\
\hline & Negative finding & Positive finding & & \\
\hline Negative finding & $90(68.7 \%)$ & $14(10.7 \%)$ & $104(79.4 \%)$ & ${ }^{1} p<0.000^{*}$ \\
\hline Positive finding & $8(6.1 \%)$ & 19 (14.5\%) & 27 (20.6\%) & \\
\hline Total & 98 (74.8\%) & $33(25.2 \%)$ & 131 (100\%) & \\
\hline
\end{tabular}

All values are presented as $\mathrm{N}(\%)$.

'Pearson Chi Square Test.

Sampling of subgigival plaque by sterile paper points

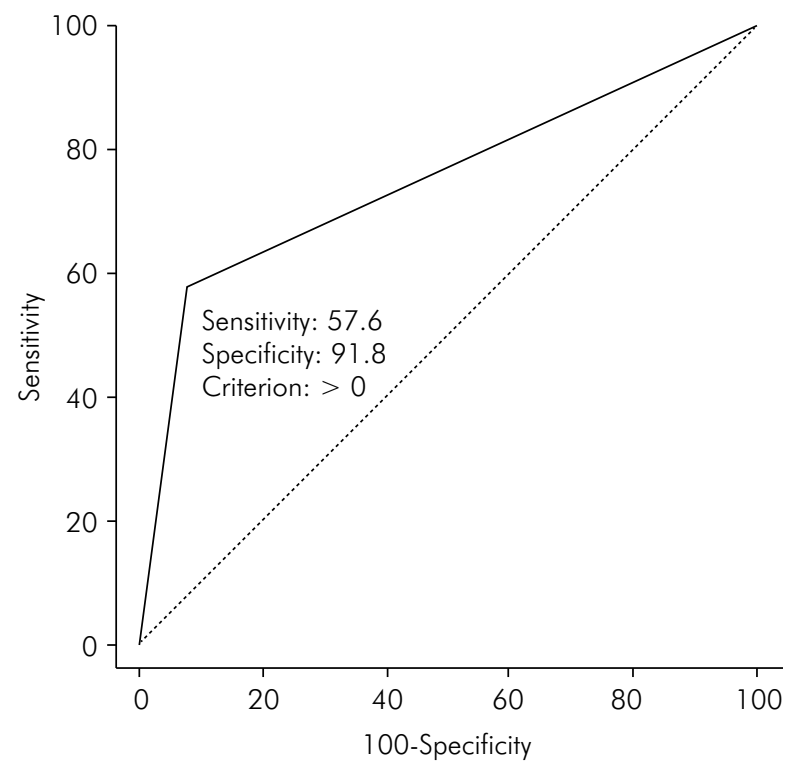

Figure 2. ROC curve for sampling methods of subgingival plaque by sterile curette and by sterile paper points.

Statistical differences of yast presence were not observed between groups or between diabetics vs. non-diabetics. Sardi et al..$^{20}$ found differences in subgingival findings of yeasts between well-controlled insulin dependent T2D patients and control group of healthy $\mathrm{CP}$ patients. When comparing the prevalence of subgingival positive findings in good vs. poorly controlled diabetics, Melton et al. ${ }^{4}$ found no significant differences. Studies examining the prevalence of subgingival yeasts in healthy patients according to their periodontal status, demonstrated the impact of the periodontal probing depth on the presence of subgingival yeasts ${ }^{8}$ which was not the case in our study. Candida spp. is an opportunistic pathogen, and it is considered as marker of immunocompromised patients. Periodontitis itself has been recognized as a state of disturbed cellular and humoral immune local response $\mathrm{e}^{23}$ and patients with diagnosed diabetes are also considered immunocompromised. Our results, which show a similar prevalence of subgingival yeasts in healthy patients and healthy patients with diagnosed $\mathrm{CP}$, are in contrast with these facts. We examined only the presence of yeasts which does not always lead to clinical infection. Some authors indicate that the presence of yeasts in subgingival sites is transient. ${ }^{24}$ The reaction of host immunity around yeasts was not a subject of investigation or the exact species of Candida genus. Candida spp. is capable of adhering to epithelial cells and inducing inflammation. ${ }^{25}$

There is an increasing number of studies investigating subgingival prevalence of Candida spp. Some studies used a sterile curette as sampling method, ${ }^{7,24}$ while others used sterile paper points. ${ }^{8,9}$ To the best of our knowledge, there is no explanation in any of these studies about the choice of the sampling method. In an attempt to answer this question, we used both methods in the same pocket and ROC analysis was carried out. A sterile 
curette method was defined as the "golden standard". Specificity, which represents true negative results, was excellent (0.919) but sensitivity, which represents the probability that test results will be positive when the disease is present, was 0.576 . The area under the curve shows a fair accuracy of the test. According to this ROC analysis, it may be concluded that subgingival plaque sampling by means of a sterile curette is more accurate than sampling by means of sterile paper points. This is in agreement with the fact that Candida spp. forms its colonies on the surface of the epithelial cells ${ }^{26}$, i.e. it is necessary to "scratch" with the help of a curette in order to ensure the accuracy of the results. On the other hand, sampling by sterile paper points is more appropriate if it is necessary to quantify Candida spp. Sampling with paper point can be standardized in terms of paper point size, duration of the presence of the paper point in the pocket and paper point pressure in the pocket. In different studies, there are differences in paper point size and insertion duration when sampling the subgingival plaque. Considering that numerous studies use paper points as sampling method, methodology should be standardized in order to compare results.

It has already been proven that subgingival sites may be a reservoir of Candida spp. ${ }^{9}$ In our study $12.9 \%$ of all patients harbored yeast in subgingival sites with no presence on tongue. The subgingival area is beneficial for Candida growth. ${ }^{16}$

\section{References}

1. Association Diabetes Association. Standards of medical care in diabetes-2013. Diabetes Care. 2013;36(Suppl 1):S11-S66.

2. Löe H. Periodontal Disease: the sixth complication of diabetes mellitus. Diabetes Care. 1993 Jan;16(1):329-34.

3. Lalla E, Cheng B, Lal S, Kaplan S, Softness B, Greenberg E, et al. Diabetes mellitus promotes periodontal destruction in children. J Clin Periodontol. 2007 Apr;34(4):294-8.

4. Melton JJ, Redding SW, Kirkpatrick WR, Reasner CA, Ocampo GL, Venkatesh A, et al. Recovery of Candida dubliniensis and other Candida species from the oral cavity of subjects with periodontitis who had well-controlled and poorly controlled type 2 diabetes: a pilot study. Spec Care in Dentist. 2010 Nov-Dec;30(6):230-4.

5. Willis A, Coulter W, Sullivan D, Coleman D, Hayes J, Bell $\mathrm{P}$, et al. Isolation of $\mathrm{C}$. dubliniensis from insulin-using
The detection of yeast prevalence and their quantification, as well as the recognition of different species, virulence factors and drug resistance in subgingival biofilm are important because of the emerging usage of antibiotics as adjuvant periodontitis therapy. The usage of a broad spectrum antibiotics may lead to Candida opportunistic infections and periodontal destruction. Some authors indicate that the presence of yeasts in the subgingival area is transient, ${ }^{24}$ i.e. the study to be carried out should be rather longitudinal than cross-sectional.

\section{Conclusions}

Candida spp. is more prevalent on tongue in the case of diabetics than in the healthy control group, regardless of periodontal status. In addition to that, diabetics with poor glycoregulation exhibited more yeast than patients with good metabolic control. The subgingival area may represent reservoir of commensals. However, longitudinal studies are needed to confirm these results. Correspondingly, it is necessary to standardize sampling methods for the collection of subgingival plaque.

\section{Acknowledgment}

This study was funded by a Project of the Ministry of Education, Science and Technological Development of the Republic of Serbia [41008].

diabetes mellitus patients. J Oral Pathol Med. 2000 Feb;29(2):86-90.

6. Khosravi A, Yarahmadi S, Baiat M, Shokri H, Pourkabireh $M$. Factors affecting the prevalence of yeasts in the oral cavity of patients with diabetes mellitus. J Mycol Med. 2008 Jun;18(2):83-8.

7. Hammad MM, Darwazeh AMG, Idrees MM. The effect of glycemic control on Candida colonization of the tongue and the subgingival plaque in patients with type II diabetes and periodontitis. Oral Surg Oral Med Oral Pathol Oral Radiol. 2013 Sep;116(3):321-6.

8. Canabarro A, Valle C, Farias M, Santos F, Lazera M, Wanke B. Association of subgingival colonization of Candida albicans and other yeasts with severity of chronic periodontitis. J Periodontal Res. 2013 Aug:48(4):428-32. doi: 10.1111/jre.12022. Epub 2012 Nov 8. 
9. Gaetti-Jardim Júnior E, Nakano V, Wahasugui TC, Cabral FC, Gamba R, Avila-Campos MJ. Occurrence of yeasts, enterococci and other enteric bacteria in subgingival biofilm of HIV-positive patients with chronic gingivitis and necrotizing periodontitis. Braz J Microbiol. 2008 Apr;39(2):257-61.

10. Al Mubarak S, Robert AA, Baskaradoss JK, Al-Zoman K, Al Sohail A, Alsuwyed A, et al. The prevalence of oral Candida infections in periodontitis patients with type 2 diabetes mellitus. J Infect Public Health. 2013 Aug;6(4):296-301.

11. Järvensivu A, Hietanen J, Rautemaa R, Sorsa T, Richardson M. Candida yeasts in chronic periodontitis tissues and subgingival microbial biofilms in vivo. Oral Dis. 2004 Mar;10(2):106-12.

12. Grenier G, Gagnon G, Grenier D. Detection of herpetic viruses in gingival crevicular fluid of patients suffering from periodontal diseases: prevalence and effect of treatment. Oral Microbiol Immunol. 2009 Dec;24(6):506-9.

13. Scully C. Oral and maxillofacial medicine: the basis of diagnosis and treatment. 2th ed: Elsevier Health Sciences; 2008. 393 p.

14. Armitage GC. Development of a classification system for periodontal diseases and conditions. Ann Periodontol. 1999 Dec;4(1):1-6.

15. Savage A, Eaton KA, Moles DR, Needleman I. A systematic review of definitions of periodontitis and methods that have been used to identify this disease. J Clin Periodontol. 2009 Jun;36(6):458-67.

16. Sardi JCO, Almeida AMF, Mendes Giannini MJS. New antimicrobial therapies used against fungi present in subgingival sites-a brief review. Arch Oral Biol. 2011 Oct;56(10):951-9.

17. Barros LM, Boriollo MFG, Alves ACBA, Klein MI, Gonçalves RB, Höfling JF. Genetic diversity and exoenzyme activities of Candida albicans and Candida dubliniensis isolated from the oral cavity of Brazilian periodontal patients. Arch Oral Biol. 2008 Dec;53(12):1172-8.

18. Epstein JB, Truelove EL, Izutzu KT. Oral candidiasis: pathogenesis and host defense. Rev Infect Dis. 1984 Jan-Feb;6(1):96-106.

19. Yang Y-L. Virulence factors of Candida species. J Microbiol Immunol Infect. 2003 Dec;36(4):223-8.

20. Sardi JCO, Duque C, Camargo GACG, Hofling JF, Gonçalves RB. Periodontal conditions and prevalence of putative periodontopathogens and Candida spp. in insulindependent type 2 diabetic and non-diabetic patients with chronic periodontitis-a pilot study. Arch Oral Biol. 2011 Oct;56(10):1098-105.

21. Belazi M, Velegraki A, Fleva A, Gidarakou I, Papanaum L, Baka D, et al. Candidal overgrowth in diabetic patients: potential predisposing factors. Mycoses. 2005 May;48(3):192-6.

22. Tapper-Jones L, Aldred M, Walker D, Hayes T. Candidal infections and populations of Candida albicans in mouths of diabetics. J Clin Pathol. 1981 Jul;34(7):706-11.

23. Murray PA. Periodontal diseases in patients infected by human immunodeficiency virus. Periodontol 2000. 1994 Oct;6(1):50-67.

24. Hintao J, Teanpaisan R, Chongsuvivatwong V, Ratarasan C, Dahlen G. The microbiological profiles of saliva, supragingival and subgingival plaque and dental caries in adults with and without type 2 diabetes mellitus. Oral Microbiol Immunol. 2007 Jun;22(3):175-81.

25. Hube B, Naglik J. Candida albicans proteinases: resolving the mystery of a gene family. Microbiology. 2001 Aug;147(8):1997-2005.

26. Hazen K. Participation of yeast cell surface hydrophobicity in adherence of Candida albicans to human epithelial cells. Infect Immun. 1989 Jul;57(7):1894-900. 